\title{
ACAROLOGY
}

\section{Suscetibilidade de Acarophenax lacunatus (Cross \& Krantz) (Prostigmata: Acarophenacidae) ao Enxofre}

\author{
José R. Gonçalves ${ }^{1}$, Lêda R.D’A. Faroni ${ }^{2}$, Raul N.C. Guedes ${ }^{1}$, Ramon M. Silva ${ }^{2}$ E \\ Flávia M. Garcia ${ }^{2}$ \\ ${ }^{1}$ Depto. Biologia Animal,goncalves_mip@hotmail.com; ${ }^{2}$ Departamento de Engenharia Agrícola \\ Univ. Federă de Viçosa, 36570-000, Viçosa, MG
}

Neotropical Entomology 36(1):112-116 (2007)

Susceptibility of Acarophenax lacunatus (Cross \& Krantz) (Prostigmata: Acarophenacidae) to Sulfur

\begin{abstract}
The parasitic mite Acarophenax lacunatus (Cross \& Krantz) is a potentially important biocontrol agent of stored grain insect pests, but its presence in insect mass rearing is undesirable. This study was carried out to evaluate the susceptibility of $A$. lacunatus to sulfur. The experimental units were petri dishes containing $30 \mathrm{~g}$ of whole wheat grains infested with 30 adults of Rhyzopertha dominica (Fabricius) (Coleoptera: Bostrichidae). The treatments consisted of pulverizing the wheat grains with sulfur, at $0,0.15,0.3,0.6,0.9$ and $1.2 \mathrm{mg}$ a.i./g, in five replicates. Three inoculations of $A$. lacunatus were carried out in each petri dish at five, ten and fifteen days after infestation with $R$. dominica. The petri dishes were stored for 60 days at $30 \pm 1^{\circ} \mathrm{C}, 60 \pm 5 \%$ R.H. and $24 \mathrm{~h}$ scotophase. The parasitic mite was susceptible to all sulfur doses used in the test. The number of eggs of $R$. dominica parasitized by A. lacunatus showed a decreasing trend with the increase in doses of sulfur, as did the number of physogastric females of the mite. The mite showed a negative correlation of their instantaneous rate of increase with sulfur dose. No mites developed at sulfur doses higher than $0.3 \mathrm{mg}$ a.i./g. The susceptibility of $A$. lacunatus to sulfur is an important aspect to consider in the control of this parasitic mite in colonies of $R$. dominica. In addition, this control method has low cost and is easy to use.
\end{abstract}

KEY WORDS: Acari, stored grain, mass rearing, Rhyzopertha dominica, acaricide

RESUMO - O ácaro parasita Acarophenax lacunatus (Cross \& Krantz) pode ser um importante agente de controle biológico de insetos-praga de grãos armazenados, mas sua presença em criações massais desses organismos é prejudicial. O objetivo deste trabalho foi avaliar a susceptibilidade desse parasita ao enxofre. As unidades experimentais consistiram de placas de Petri, contendo $30 \mathrm{~g}$ de grãos de trigo infestados com 30 adultos de Rhyzopertha dominica (Fabricius) (Coleoptera: Bostrichidae). Os tratamentos consistiram no polvilhamento dos grãos de trigo com enxofre, nas doses correspondentes a $0 ; 0,15 ; 0,30 ; 0,60 ; 0,90$ e 1,20 mg i.a./g, em cinco repetições. Foram realizadas três inoculações de $A$. lacunatus por placa de Petri, cinco, dez e quinze dias depois da infestação de $R$. dominica. Em seguida, as placas foram armazenadas por 60 dias a $30 \pm 1{ }^{\circ} \mathrm{C}, 60 \pm 5 \%$ UR e escotofase de $24 \mathrm{~h}$. O ácaro parasita mostrou-se susceptível a todas doses de enxofre avaliadas. O número de ovos de $R$. dominica parasitados por $A$. lacunatus apresentou uma tendência decrescentecom o aumento das doses do acaricida, assim como o número de fêmeas fisogástricas do ácaro. A taxa instantânea de crescimento de $A$. lacunatus se reduziu com o aumento das doses de enxofre, não ocorrendo o desenvolvimento do parasita nas doses superiores a $0,30 \mathrm{mg}$ i.a./g. A suscetibilidade de A. lacunatus ao enxofre é importante, uma vez que não existe na literatura uma alternativa de controle desse parasita sobre criações de insetos. Além disso, esse método de controle apresenta baixo custo e é de fácil utilização.

PALAVRAS-CHAVE: Acari, grãos armazenados, criação massal, Rhyzopertha dominica, acaricida

O ácaro Acarophenax lacunatus (Cross \& Krantz) vem se destacando como um agente potencialmente útil para o controle biológico de coleópteros-praga de grãos armazenados (Oliveira et al. 2003). O ácaro parasita os ovos de seus hospedeiros em temperaturas de $18^{\circ} \mathrm{C}$ a $40^{\circ} \mathrm{C}$, não permitindo o seu desenvolvimento (Faroni et al. 2001).
A temperatura ótima para $A$. lacunatus é $30^{\circ} \mathrm{C}$, chegando a suprimir $90 \%$ dos ovos de Rhyzopertha dominica (Fabricius) (Coleoptera: Bostrichidae), no período de 23 dias após sua inoculação sobre populações de 25 adultos do coleóptero (Gonçalves et al. 2001).

O primeiro relato de $A$. lacunatus interagindo com praga 
de grãos armazenados foi feito por Cross \& Krantz (1964), que o coletaram em adultos do coleóptero Cryptolestes ferrugineus (Stephens) (Coleoptera: Cucujidae). Estudos mais aprofundados de $A$. lacunatus iniciaram-se com a descoberta por Faroni et al. (2000) desse parasita sobre $R$. dominica. Trabalhos realizados por Oliveira et al. (2002, 2003) constataram que esse ácaro é capaz de parasitar ovos dos coleópteros C. ferrugineus, Dinoderus minutus (Fabricius) (Bostrichidae) e Tribolium castaneum (Herbst) (Tenebrionidae).

O hospedeiro em que $A$. lacunatus melhor se desenvolve é $R$. dominica, podendo alcançar até 12 gerações em um mês (Faroni et al. 2001). Um a das dificuldades da manipulação de criações massais de insetos hospedeiros é que dificilmente elas estão livres desse ácaro. Esse problema também é observado para o ácaro Acarophenax tribolii Newstead \& Duvall sobre Tribolium confusum Duval e T. castaneum (Trivelli \& Velázquez 1985, Arnaud et al. 1996) e para Pyemotes tritici (Lagrèze-Fossat \& Montané) (Acari: Pyemotidae) sobre Sitotroga cerealella (Olivier) (Lepidoptera: Gelechidae), Acanthoscelides obtectus Say (Coleoptera: Bruchidae) e Phoracantha semipunctata Fabricius (Coleoptera: Cerambycidae) (Navarro et al. 1983, Trivelli \& Velázquez 1985, Hanks et al. 1992).

A dificuldade em se manter criações de insetos livres de ácaros também vem sendo observada em colônias de Apis cerana Fabricius, Apis mellifera Linnaeus e Melipona colimana Ayala (Hymenoptera: Apidae), que são atacadas por Varroa jacobsoni (Oudemans), Varroa destructor Anderson \& Trueman (Acari: Varroidae) e P. tritici (De Jong et al. 1982, Anderson \& Trueman 2000).

$\mathrm{Na}$ tentativa de controlar a infestação de ácaros em criações massais de insetos, vários acaricidas já foram avaliados, principalmente contra Pyemotes spp. (Navarro et al. 1983, Dinabandhoo \& Dogra 1984), uma vez que esses ácaros também causam dermatites em seres humanos (Rosen et al. 2002). Estudos realizados por Hanks et al. (1992) constataram que o uso do enxofre reduziu a densidade de $P$. tritici e, conseqüentemente, aumentou a emergência de $P$. semipunctata. Já Arnaud et al. (1996), na busca de uma alternativa para eliminar o ácaro $A$. tribolii de criações massais de $T$. castaneum, pulverizaram os adultos desse coleóptero com uma solução de $5 \%$ de formol e obtiveram mortalidade dos ácaros após um período de $10 \mathrm{~h}$ de exposição. No presente estudo, objetivou-se avaliar o efeito de diferentes doses de enxofre sobre o potencial de parasitismo de $A$. lacunatus, visando a obtenção de criações massais de $R$. dominica livres da presença do ácaro parasita.

\section{Material e Métodos}

Criação de $\boldsymbol{R}$. dominica. O coleóptero $R$. dominica foi criado em câmara climatizada, à temperatura de $30 \pm$ $1{ }^{\circ} \mathrm{C}, 60 \pm 5 \%$ UR e escotofase de $24 \mathrm{~h}$, a partir de adultos provenientes da criação estoque do laboratório. A criação foi iniciada com 50 adultos, em placas de Petri contendo grãos de trigo (Triticum aestivum L.) previamente expurgados, com teor de água de $13 \%$ b.u. (base úmida). Sete dias após o início da criação, tempo suficiente para que os insetos iniciassem a oviposição, Os ovos da praga foram coletados com peneira de orifícios de $1 \mathrm{~mm}$ de diâmetro e colocados em outras placas com a mesma dieta. Os ovos foram coletados de acordo com um calendário, para que houvesse disponibilidade de adultos de $R$. dominica com idade conhecida (Faroni et al. 2001).

Criação de A. lacunatus. Espécimes de A. lacunatus foram obtidos de criações massais de $R$. dominica infestadas por esse parasita há mais de quatro anos em laboratório. $\mathrm{O}$ ácaro foi criado em populações de $R$. dominica a $30 \pm 1{ }^{\circ} \mathrm{C}, 60 \pm$ $5 \%$ UR e escotofase de $24 \mathrm{~h}$, porém em salas diferentes das criações de $R$. dominica isentas do ácaro, para evitar a contaminação (Faroni et al. 2001).

Exposição de A. lacunatus ao enxofre. As unidades experimentais consistiram de placas de Petri de $140 \times 10 \mathrm{~mm}$ (diâmetro x altura), contendo $30 \mathrm{~g}$ de grãos de trigo com teor de água de $13 \%$ b.u. (base úmida), infestados com 30 adultos não-sexados de $R$. dominica, com idade entre três e sete dias. Os tratamentos consistiram no polvilhamento com enxofre em pó (grau de pureza 99\%) em diferentes doses sobre grãos de trigo, correspondentes a $0 ; 0,15 ; 0,30 ; 0,60 ; 0,90$ e 1,20 $\mathrm{mg}$ do ingrediente ativo (i.a.)/g, em cinco repetições. Foram realizadas três inoculações de cinco fêmeas adultas de $A$. lacunatus por placa de Petri, cinco, dez e quinze dias após a infestação de $R$. dominica, período suficiente para que a praga colocasse os primeiros ovos. As placas foram revestidas com filme plástico de PVC para evitar que os insetos e os ácaros escapassem e também para prevenir contaminação por outras espécies. Todos os tratamentos foram armazenados por 60 dias em câmara climatizada ajustada a $30 \pm 1{ }^{\circ} \mathrm{C}, 60 \pm 5 \%$ UR e escotofase de $24 \mathrm{~h}$.

Após o período de armazenamento avaliaram-se as populações de $R$. dominica inoculadas com o ácaro e a massa de grãos de trigo presentes por placa. Para isso, a massa de grãos de cada placa foi passada em peneira com orifícios de $1 \mathrm{~mm}$ de diâmetro, separando-se os insetos dos grãos e o resíduo (pó) contendo fases imaturas (ovos e larvas) de $R$. dominica e ácaros. Em seguida, procedeu-se à determinação da perda de massa dos grãos de trigo (\%), através da alteração da massa com o término do experimento, e à contagem do número de insetos adultos de $R$. dominica. Para quantificar o número de ovos de $R$. dominica parasitados ou não por $A$. lacunatus, de fêmeas do ácaro em processo de fisogastria e de larvas de $1^{\circ}$ ínstar de $R$. dominica, foi analisado o resíduo da massa de grãos com auxílio de microscópio estereoscópico.

A taxa instantânea de crescimento de A. lacunatus $\left(\mathrm{r}_{\mathrm{i}}\right)$ foi calculada usando a equação: $\mathrm{r}_{\mathrm{i}}=\left[\ln \left(\mathrm{N}_{\mathrm{f}} / \mathrm{N}_{0}\right] / \Delta \mathrm{t}\right.$; onde $\mathrm{N}_{\mathrm{f}}=$ número final de ácaros vivos; $\mathrm{N}_{0}=$ número inicial de ácaros vivos; $\Delta \mathrm{t}=$ variação de tempo (número de dias em que o ensaio foi executado) (Walthall \& Stark 1997).

Os dados avaliados foram submetidos à análise de regressão, com exceção da taxa instantânea de crescimento $\left(\mathrm{r}_{\mathrm{i}}\right)$ de $A$. lacunatus e da perda de massa dos grãos de trigo cujas médias foram comparadas pelo teste Duncan, a 5\% de significância. 


\section{Resultados e Discussão}

O ácaro A. lacunatus mostrou-se susceptível a todas as doses de enxofre. O número de ovos de $R$. dominica parasitados por $A$. lacunatus apresentou uma tendência decrescente com o aumento da dose do acaricida $\left(\mathrm{F}_{2,27}=\right.$ 257,75; $\mathrm{P}<0,001$ ) (Fig. 1). Essa tendência também foi observada para o número de fêmeas fisogástricas de $A$. lacunatus $\left(\mathrm{F}_{2,27}=100,93 ; \mathrm{P}<0,001\right)$ (Fig. 1). O parasita não sobreviveu nas doses acima de $0,30 \mathrm{mg}$ i.a./g (Fig. 1). Resultados semelhantes foram observados por Hanks et al. (1992), quando utilizaram $500 \mathrm{mg}$ de enxofre sobre o ácaro P. tritici. O uso do enxofre na prevenção e/ou controle de ácaros é uma prática que já vem sendo empregada há vários anos em condições de campo (Jeppson et al. 1975). Em criações massais de insetos, o acaricida vem se destacando, uma vez que pode conferir proteção contra o ataque de ácaros e não afetar a longevidade e fecundidade dos insetos (Hanks et al. 1992).

As dificuldades para se prevenir infestação de ácaros em criações massais de insetos são decorrentes da inexistência de produtos registrados para esse fim. No entanto, pesquisadores vêm tentando adaptar produtos recomendados para outras espécies de ácaros em culturas ou em produtos armazenados para o controle desses organismos em criações de insetos (Dinabandhoo \& Dogra 1984, Hanks et al. 1992).

Uma vez notada a infestação de ácaros em criações de insetos em laboratório, seu controle é praticamente impossível, sendo, às vezes, necessário descartar tais criações. Com isso, a prevenção da infestação é de suma importância (Flechtmann \& Zem 2002). Algumas espécies de ácaros que atacam colônias de insetos em laboratório podem ser controladas através da limpeza e higienização das salas de criação, dos equipamentos e de materiais utilizados para multiplicação desses organismos, uma vez que podem existir focos de infestação de ácaros nesses locais (Navarro 1993).

Doses de enxofre inferiores a 0,30 mg i.a./g apresentaram efeito subletal no ácaro A. lacunatus. Isso foi constatado através da tendência decrescente do número de fêmeas fisogástricas do ácaro e do número de ovos de $R$. dominica parasitados, com o aumento da dose de 0,15 para 0,30 mg i.a./g. O efeito subletal também foi observado para $P$. tritici, quando, após a aplicação do enxofre, o número médio de ácaros infestando $P$. semipunctata foi reduzido significativamente de $39 \pm 12$ para $2,9 \pm 1,2$ (Hanks et al. 1992). Existem evidências de que o enxofre também causa efeitos subletais sobre espécies de Trichogramma (Hymenoptera: Trichogrammatidae) e Podisus nigrispinus (Dallas) (Hemiptera: Pentatomidae), os quais apresentam, respectivamente, seu potencial de parasitismo e de predação reduzido com a utilização do acaricida (Thomson et al. 2000, Torres et al. 2002).

A taxa instantânea de crescimento de A. lacunatus também apresentou diferença significativa com a utilização do enxofre $\left(\mathrm{F}_{5,24}=144,34 ; \mathrm{P}<0,001\right)$ (Fig. 2). Nas doses acima de 0,30 mg i.a./g, não houve desenvolvimento do parasita. Porém, A. lacunatus se desenvolveu nas doses de 0,15 e $0,30 \mathrm{mg}$

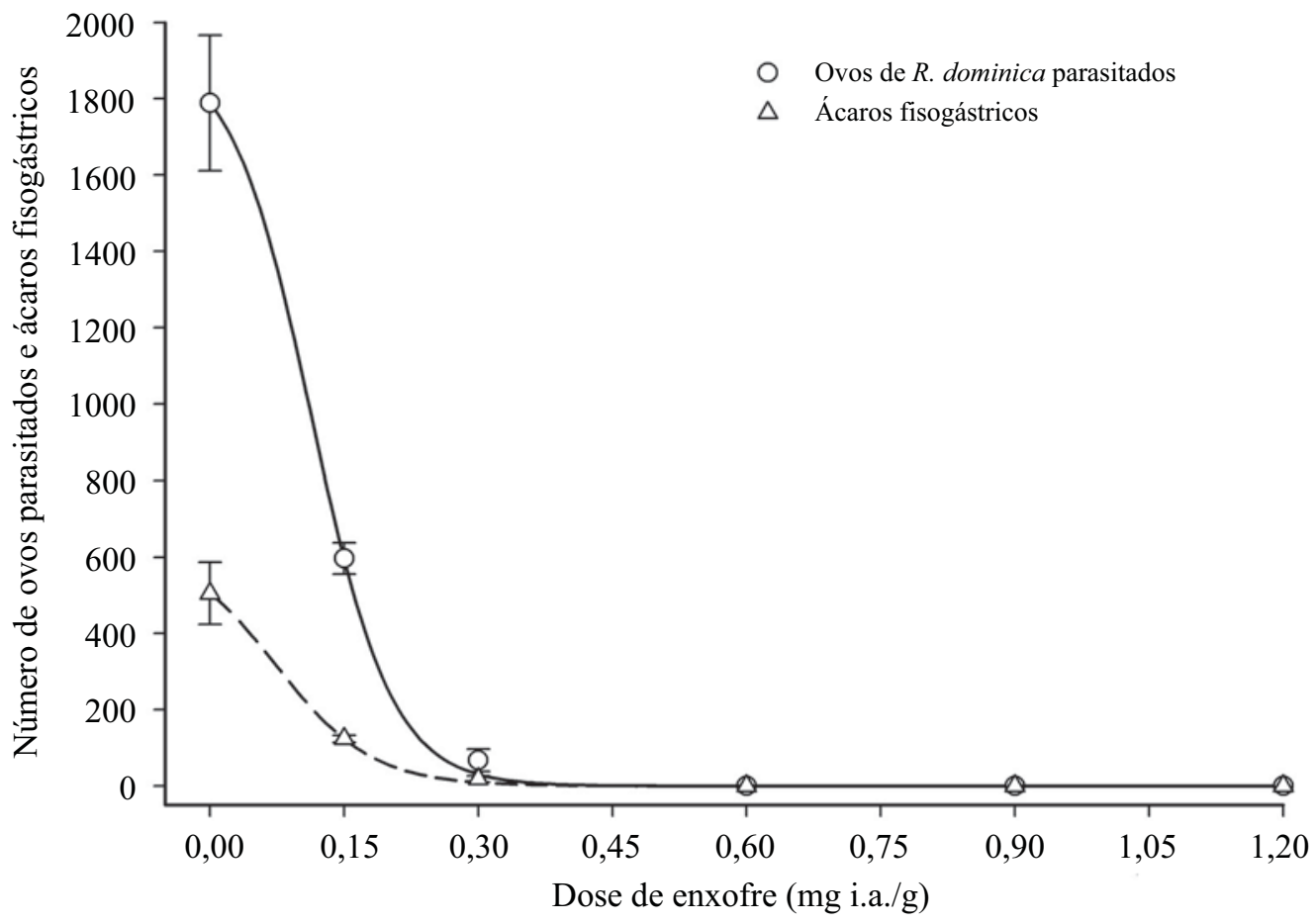

Fig. 1. Número de ovos de $R$. dominica parasitados e fêmeas fisogástricas de A. lacunatus em função de doses de enxofre misturado aos grãos de trigo, após 60 dias de armazenamento a $30 \pm 1{ }^{\circ} \mathrm{C}, 60 \pm 5 \%$ de UR e $24 \mathrm{~h}$ de escotofase. ([-] y $=$ $\left.1933,27 /(1+\exp \cdot(-(\mathrm{x}-0,11) /-0,04)) ; \mathrm{F}_{2,27}=257,75 ; \mathrm{P}<0,001 ; \mathrm{R}^{2}=0,95\right)\left([---] \mathrm{y}=627,22 /(1+\exp (-(\mathrm{x}-0,08) /-0,05)) ; \mathrm{F}_{2,27}=100,93\right.$; $\left.\mathrm{P}<0,001 ; \mathrm{R}^{2}=0,88\right)$ 


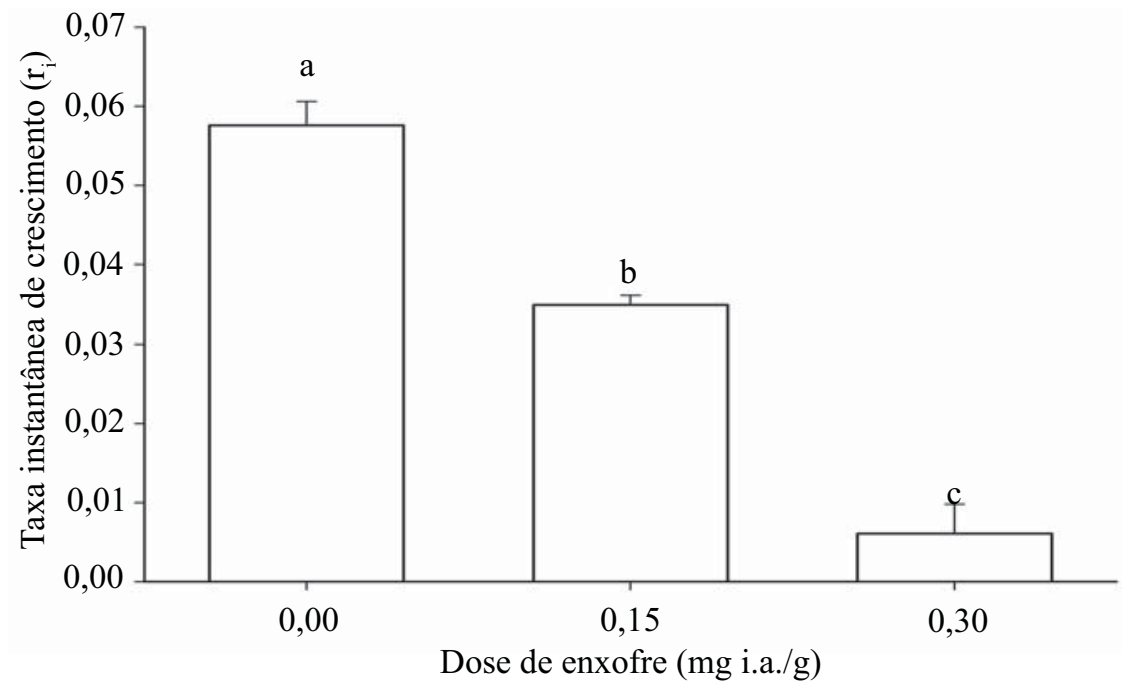

Fig. 2. Taxa instantânea de crescimento de A. lacunatus, após 60 dias de armazenamento a $30 \pm 1{ }^{\circ} \mathrm{C}, 60 \pm 5 \%$ de UR e $24 \mathrm{~h}$ de escotofase, em função de doses de enxofre misturado aos grãos de trigo. As médias seguidas pela mesma letra não diferem entre si, pelo teste de Duncan, a 5\% de significância.

i.a./g do acaricida, com a taxa instantânea de crescimento (r.) de $0,035 \pm 0,001$ e $0,006 \pm 0,004$, respectivamente (Fig. 2). Resultados semelhantes foram observados por Gonçalves et al. (2004) no desenvolvimento de A. lacunatus sobre grãos de trigo tratados com bifentrina. Essa resistência pode estar relacionada ao elevado número de gerações do ácaro num curto período de tempo (12 gerações mensais a $\left.30^{\circ} \mathrm{C}\right)$ (Faroni et al. 2001).

$\mathrm{O}$ número de ovos $\left(\mathrm{F}_{1,28}=339,56 ; \mathrm{P}<0,001\right)$ e larvas $\left(\mathrm{F}_{1,28}=241,40 ; \mathrm{P}<0,001\right)$ de $R$. dominica apresentou uma tendência crescente com o aumento das doses de enxofre (Fig. 3). Isto se deve ao potencial de parasitismo de ovos de $R$. dominica pelo ácaro A. lacunatus, nas menores doses do acaricida.

Não houve diferença significativa em relação ao número de adultos de $R$. dominica $\left(\mathrm{F}_{5,24}=1,51 ; \mathrm{P}=0,23\right)$, porém, foi observada diferença significativa para a perda de massa dos grãos de trigo $\left(\mathrm{F}_{5,24}=6,71 ; \mathrm{P}<0,001\right)$, embora a menor perda só tenha ocorrido na ausência do acaricida.

$\mathrm{Na}$ ausência do enxofre, A. lacunatus proporcionou

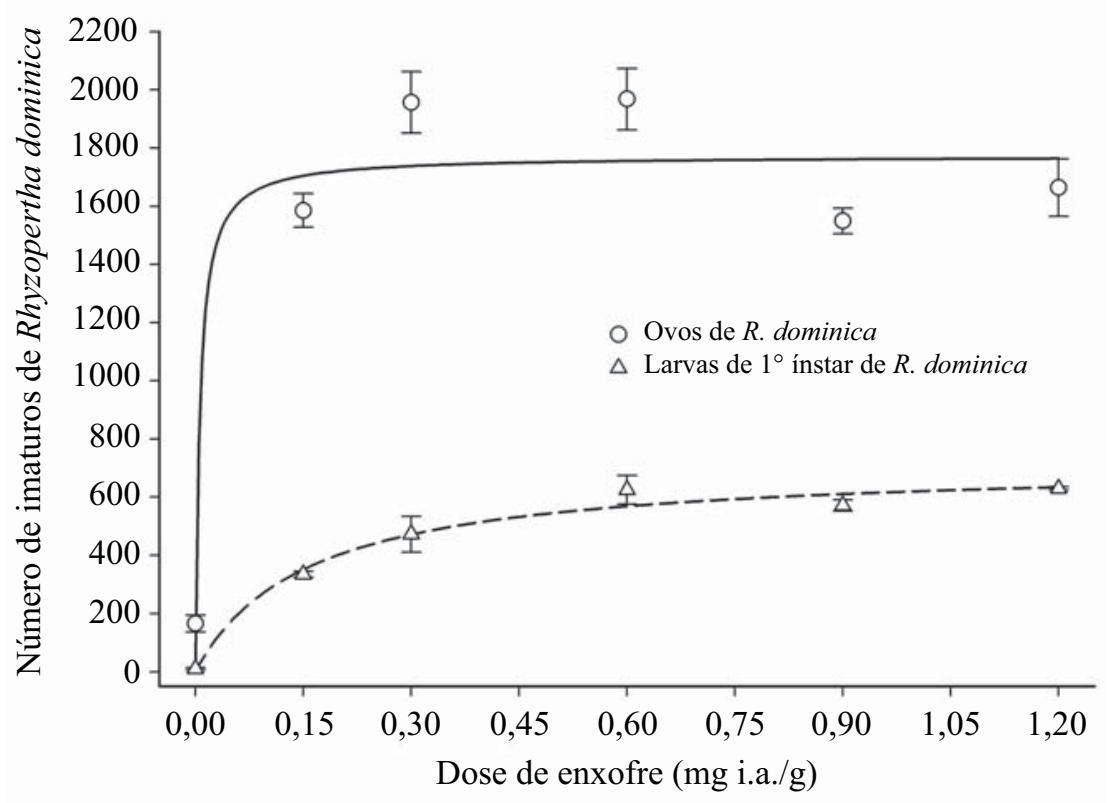

Fig. 3. Efeito de doses de enxofre misturado aos grãos de trigo sobre o potencial de A. lacunatus na redução do número de ovos e larvas de $R$. dominica, após 60 dias de armazenamento a $30 \pm 1^{\circ} \mathrm{C}, 60 \pm 5 \%$ de UR e $24 \mathrm{~h}$ de escotofase. $([-]$ y $=1787,73$. $\left.\mathrm{x} /(0,55+\mathrm{x}) ; \mathrm{F}_{1,28}=339,55 ; \mathrm{P}<0,001 ; \mathrm{R}^{2}=0,92\right) ;\left([---] \mathrm{y}=716,93 \times \mathrm{x} /(5,20+\mathrm{x}) ; \mathrm{F}_{2,28}=241,40 ; \mathrm{P}<0,001 ; \mathrm{R}^{2}=0,90\right)$. 
os menores números de ovos e larvas de $R$. dominica e, conseqüentemente, a menor perda de massa dos grãos de trigo. Nas doses de 0,90 a 1,20 mg i.a./g de enxofre, também se reduziu o número de ovos de $R$. dominica. Essa redução pode ter sido ocasionada pelo uso do acaricida, uma vez que nessa faixa de dose, A. lacunatus não sobreviveu. Este fato foi relatado por Ware (1994), ao mencionar que o enxofre, embora seja um acaricida, pode afetar o desenvolvimento dos insetos.

Neste estudo, portanto, A. lacunatus se mostrou susceptível ao enxofre. Esse resultado é relevante, uma vez que não existe na literatura uma alternativa para o controle desse parasita em criações massais de insetos. Todavia, altas doses desse acaricida também podem afetar o desenvolvimento do hospedeiro. Estudos utilizando $R$. dominica associada a diferentes doses de enxofre, sem o ácaro, devem ser realizados com o intuito de se obter a dose do acaricida que elimine o parasita e não influencie a biologia do hospedeiro.

\section{Referências}

Anderson, D.L. \& J.W.H. Trueman. 2000. Varroa jacobsoni (Acari: Varroidae) is more than one species. Exp. Appl. Acarol. 24: 165-189.

Arnaud, L., J. Mignon, J.C. Gilson \& E. Haubruge. 1996. A simple technique to relieve Tribolium castaneum (Coleoptera: Tenebrionidae) of Acarophenax tribolii (Acarina: Pyemotidae), p.86-87. In Material contributed by workers on Tribolium and other coleopteran. San Bernadino, California, School of Natural Sciences, California State University. 253p. (Information Bulletin)

Cross, E.A. \& G.W. Krantz. 1964. Two new species of the genus Acarophenax Newstead and Durvall 1918 (Acarina: Pyemotidae). Acarologia 6: 287-295.

Dinabandhoo, C.L. \& G.S. Dogra. 1984. Incidence and control of ectoparasitic mite, Pyemotes herfsi Oudenmans of the Indian honeybee, Apis cerana Fab. Am. Bee J. 120: 44-47.

Faroni, L.R.A.; Guedes, R.N.C. ; Matioli, A.L.. 2000. Potential of Acarophenax lacunatus (Prostigmata: Acarophenacidae) as a biological control agent of Rhyzopertha dominica (Coleoptera: Bostrichidae). J. Stored Prod. Res., 36: 55-63.

Faroni, L.R.A., R.N.C. Guedes \& A.L. Matioli. 2001. Effect of temperature on development and population growth of Acarophenax lacunatus (Cross \& Krantz) (Prostigmata: Acarophenacidae) on Rhyzopertha dominica (F.) (Coleoptera: Bostrichidae). Biocontrol Sci. Tech. 11: 7-14.

Flechtmann, C.H.W. \& A.C. Zem. 2002. Ácaros de produtos armazenados, p.807-856. In I. Lorini, L.H. Miike \& V.M. Scussel (eds.), Armazenagem de grãos. Campinas, Instituto Bio Geneziz. 983p.

Gonçalves, J.R., L.R.A. Faroni, R.N.C. Guedes \& C.R.F. de Oliveira. 2001. Parasitismo de Acarophenax lacunatus (Prostigmata: Acarophenacidae) em ovos de Rhyzopertha dominica (Coleoptera: Bostrichidae). Eng. Agric. 9: 242-250.
Gonçalves, J.R., L.R.A. Faroni, R.N.C. Guedes \& C.R.F. de Oliveira. 2004. Insecticide selectivity to the parasitic mite Acarophenax lacunatus (Cross \& Krantz) (Prostigmata: Acarophenacidae) on Rhyzopertha dominica (Fabr.) (Coleoptera: Bostrichidae). Neotrop. Entomol. 33: 243-248.

Hanks, L.M., J.S. McCelfresh, J.G. Millar \& T.D. Paine. 1992. Control of the straw itch mite (Acari: Pyemotidae) with sulfur in an insect rearing facility. J. Econ. Entomol. 85: 683-686.

Jeppson, L.R., H.H. Keifer \& E.W. Baker. 1975. History of chemical control and mite resistance to acaricides, p.47-61. In L.R. Jeppson, H.H. Keifer \& E.W. Baker (eds.), Mites injurious to economic plants. University of California Press, Berkley, USA, $614 \mathrm{p}$.

Jong, D.D. De, R.A. Morse \& G.C. Eickwort. 1982. Mite pests of honey bees. Annu. Rev. Entomol. 27: 229-252.

Navarro, M.A. 1993. El Trichogramma spp.: Producción, uso y manejo en Colombia, Palmira, ICA., 184p.

Navarro, R., C. Luna \& C. Juarez. 1983. Prueba de diferentes substratos para la cria masiva de Sitotroga cerealella. Agron. Trop. 33: 111-121.

Oliveira, C.R.F. de, L.R.A. Faroni, R.N.C. Guedes \& A. Pallini. 2003. Parasitism by the mite Acarophenax lacunatus on beetle pests of stored products. Biocontrol 48: 503-513.

Oliveira, C.R.F. de, L.R.A. Faroni, R.N.C. Guedes, A. Pallini \& J.R. Gonçalves. 2002. Parasitismo de Acarophenax lacunatus (Cross \& Krantz) (Prostigmata: Acarophenacidae) sobre Dinoderus minutus (Fabr.) (Coleoptera: Bostrichidae). Neotrop. Entomol. 31: 245-248.

Rosen, S., I. Yeruham \& Y. Braverman. 2002. Dermatitis in humans associated with the mites Pyemotes tritici, Dermanyssus gallinae, Ornithonyssus bacoti and Androlaelaps casalis in Israel. Med. Vet. Entomol. 16: 442-444.

Thomson, L.J., D.C. Glenn \& A.A. Hoffmann. 2000. Effects of sulfur on Trichogramma egg parasitoids in vineyards: Measuring toxic effects and establishing release windows. Australian J. Exp. Agric. 40: 1165-1171.

Torres, J.B., C.S.A. Silva-Torres, M.R. Silva \& J.F. Ferreira. 2002. Compatibilidade de inseticidas e acaricidas com o percevejo predador Podisus nigrispinus (Dallas) (Heteroptera: Pentatomidae) em algodoeiro. Neotrop. Entomol. 31: 311317.

Trivelli, H.Dell'O. \& C.J.A. Velásquez. 1985. Insectos que dañan granos y productos almacenados, FAO/RLAC, Santiago, Chile, 142p.

Walthall, W.K. \& J.D. Stark. 1997. Comparison of two population level ecotoxicological endpoints: The intrinsic $\left(\mathrm{r}_{\mathrm{m}}\right)$ and instantaneous $\left(\mathrm{r}_{\mathrm{i}}\right)$ rates of increase. Environ. Toxicol. Chem. 16: 1068-1073.

Ware, G.W. 1994. Insecticide, p.41-74. In The pesticide book, 4a ed. Fresno, CA, Thomson Publications. 386p.

Received 11/VIII/05. Accepted 23/VI/06. 